\title{
Preconditioned somatothermal stimulation on median nerve territory increases myocardial heat shock protein 70 and protects rat hearts against ischemia-reperfusion injury
}

\author{
Jen-Hwey Chiu, MD, PhD, ${ }^{\mathrm{a}, \mathrm{b}}$ Meng-Ting Tsou, MD, ${ }^{\mathrm{a}, \mathrm{c}}$ Hwa-Hsung Tung, MS, ${ }^{\text {a }}$ Chih-Ho Tai, MB, ${ }^{a}$ \\ Shen-Kou Tsai, MD, PhD, ${ }^{d}$ Chun-Lien Chih, MS, ${ }^{e}$ Jaung-Geng Lin, MD, PhD, ${ }^{f}$ and Chew-Wun Wu, MD
}

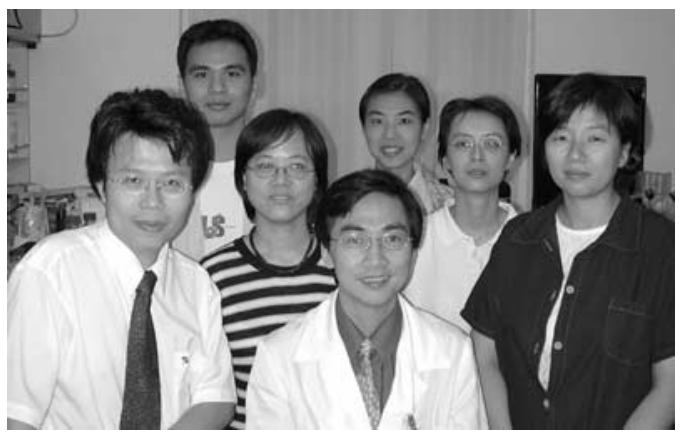

Chen, Wu, Lay, Chiu, Wang, Lin, Tsou (left to right)

Objective: This study was designed to test the hypotheses that local somatothermal stimulation on the left median nerve territory increases myocardial heat shock protein 70 and that preconditioning of rats with local somatothermal stimulation protects the hearts against subsequent ischemia-reperfusion injury.

Methods: Local somatothermal stimulation was brought about by means of application of a heating rod over and above the left median nerve territory $(1.5 \mathrm{~cm}$ proximal to the palm crease) in male Sprague-Dawley rats. After rats were treated with local somatothermal stimulation, the gene expression of heat shock protein 70 in regional muscle, heart, and liver was assessed by means of Western blotting and reverse transcription-polymerase chain reaction. In addition, durations of arrhythmia, mortality rates, and mitochondrial functions were compared between groups preconditioned with or without local somatothermal stimulation followed by subsequent myocardial ischemia-reperfusion injury.

From the Institute of Traditional Medicine, School of Medicine, National Yang-Ming University ${ }^{\mathrm{a}}$; the Division of General Surgery, Department of Surgery, Veterans General Hospital, Taipei; Taiwan, Republic of China ${ }^{\text {b }}$; the Department of Family Physicians, Mackay Memorial Hospital'; the Department of Anesthesia, School of Medicine, National Yang-Ming University ${ }^{\mathrm{d}}$; the Department of Surgery, School of Medicine, National Yang-Ming University ${ }^{\mathrm{e}}$; the Institute of Chinese Medical Science, China Medical College, ${ }^{\mathrm{f}}$ Taichung, Taiwan, Republic of China

This work was supported by grants from National Science Council, Republic of China (NSC 89-2314-B-010-014 and NSC 90-2320-B-010-054).

Received for publication Jan 4, 2002; revisions requested April 16, 2002; revisions received June 12, 2002; accepted for publication July 15, 2002.

Address for reprints: Jen-Hwey Chiu, MD, Institute of Traditional Medicine, National Yang-Ming University, No 155, Section 2, Li-Nong St, Peitou, Taipei, 112, Taiwan (E-mail: chiujh@mailsrv.ym.edu.tw).

J Thorac Cardiovasc Surg 2003;125:678-85

Copyright $\odot 2003$ by The American Association for Thoracic Surgery

$0022-5223 / 2003 \$ 30.00+0$

doi: $10.1067 / \mathrm{mtc} .2003 .29$
Results: The results showed that the gene expression of heat shock protein 70 was upregulated in the muscle beneath the area of local somatothermal stimulation, as well as in the heart, although not in the liver. When animals were preconditioned with local somatothermal stimulation on the left median nerve territory followed by subsequent ischemia-reperfusion injury of the heart, there were significant decreases of creatine kinase level from the heart, duration of arrhythmia, mortality rate, and improved mitochondrial respiratory function compared with that seen in those without local somatothermal stimulation preconditioning.

Conclusion: We conclude that local heat stress preconditioning on the left median nerve territory has a potential cardioprotective effect against subsequent ischemiareperfusion injury.

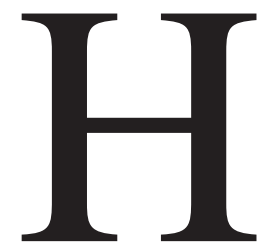

eat shock proteins (Hsps), a collective of specialized protein-Hsp chaperones, are essential cellular machineries and are induced under conditions of stress to prevent aggregation and to assist refolding of misfolded proteins. The induction of Hsps has been speculated to protect organs from ischemia-reperfusion injury, stress-induced apoptosis, and the toxic effects of nitric oxide. ${ }^{1,2} \mathrm{~A}$ number of studies suggest that the expression of Hsps by prior sublethal heat stress (hyperthermic preconditioning) attenuates the heat-induced cellular changes to a subsequent severe heat challenge. The development of thermotolerance can be initiated by preconditioning the animals not only with repeated hyperthermia but also with ischemia-reperfusion challenge or low doses of various chemical stressors. 3,4

Previous studies suggest that an inverse correlation exists between expression of Hsp70 induced by means of ischemic or thermal preconditioning and injury of many 
organs, such as the kidney, heart, and small intestine. ${ }^{5}$ By using transgenic mice, ${ }^{6,7}$ protein delivery virus vector, ${ }^{8}$ and gene-transfected rats ${ }^{9}$ as working systems, there is evidence that overexpressed Hsp70 plays an important and direct role in the enhancement of myocardial tolerance against ischemia-reperfusion injury. In most animal models, experimental heatstroke preconditioning could be achieved by exposing animals to a high ambient temperature, usually up to $41^{\circ} \mathrm{C}$, to induce Hsps in many organs. ${ }^{10}$ However, maneuvers with either tissue or whole-body hyperthermia are cumbersome and impractical in human subjects.

Recent scientific studies suggest that stimulation of the median nerve regulates the functions of the cardiovascular and autonomic systems. For example, median nerve stimulation improves left ventricular function in those patients with coronary heart disease, improves myocardial microcirculation in rabbits, and reverses the reflex-induced myocardial ischemia in cats. ${ }^{11-13}$ Nevertheless, information about how the gene expression of myocardial Hsp70 is affected by median nerve stimulation remains lacking.

Our recent works have shown that local somatothermal stimulation (LSTS), in contrast to whole-body hyperthermia, regulates the activity of the sphincter of Oddi in cats, rabbits, and human subjects, as well as the motility of the internal anal sphincter in rabbits and human subjects. In both circumstances the effects of LSTS are mediated by the activation of heat-sensitive neural release of nitric oxide. ${ }^{14,15}$ Moreover, we also demonstrate that preconditioned LSTS on the right seventh intercostal nerve territory increases hepatic Hsp70 and protects the liver from ischemiareperfusion injury in rats. ${ }^{16}$ Interestingly, the critical temperatures evoking such visceral responses are around $41^{\circ} \mathrm{C}$ to $43^{\circ} \mathrm{C}$, which are similar to those inducing Hsp expression in many studies. ${ }^{10,14-16}$ In this study 2 major questions are to be answered. First, is the myocardial gene expression of Hsp70 upregulated when local heat stress (LSTS) is applied to left median nerve territory? Second, does preconditioning the animals with LSTS protect the heart against subsequent ischemia-reperfusion injury?

\section{Methods}

\section{Animals}

Male Sprague-Dawley rats weighting 250 to $300 \mathrm{~g}$ were obtained from the animal center of the National Science Council, Taiwan, Republic of China. They were fed with standard diet and water ad libitum and treated according to the "Guide for the Care and Use of Laboratory Animals" (National Academy Press, 1996). The studies were approved by the committee of experimental animals of National Yang-Ming University.

\section{Antibody and Western Blot Analysis for Hsp Expression}

Mouse monoclonal antibody against inducible Hsp70 was purchased commercially (H53220-050, Transduction Laboratory).
Tissue homogenates from rat livers, hearts, and the muscle beneath the LSTS site were obtained in the presence of protease inhibitors on ice, and the homogenate protein was assayed by using the method of Bradford. ${ }^{17}$ Fifty micrograms of homogenate was separated on $10 \%$ sodium dodecylsulfate-polyacrylamide gel electrophoresis transferred to a nitrocellulose membrane. The membranes were blocked with skim milk and incubated with 10,000-fold diluted monoclonal antibody against Hsp70. After washing and incubation with 5000-fold diluted biotin-conjugated secondary antibody (anti-mouse IgG, horseradish peroxidase, ME5345; Transduction Laboratory), Hsp70 was detected by using enhanced chemiluminescence (Amersham Pharmacia Biotech, Inc) and analyzed with autoradiography. Monoclonal antibody against $\alpha$-tubulin was used as an internal control. The optical density values were analyzed from a computer equipped with image analysis software (PhotoCapt, Vilber Lourmat).

\section{Extraction of RNA and Reverse}

\section{Transcription-Polymerase Chain Reaction}

Total RNA was isolated from rat organs (muscle, heart, and liver) by using a modified single-step guanidinium thiocyanate method (TRI REAGENT, T-9424; Sigma). ${ }^{18}$ Reverse transcription (RT) was performed with an RT kit. One microgram of rat tissue RNA was used as a template, and RT-generated cDNAs encoding Hsp70 and glyceraldehyde-3-phosphate dehydrogenase (G3PDH; internal control) were amplified with polymerase chain reaction (PCR). The primers used in this study are Hsp70 (354 bp; sense, 5-AACGTG-CTG-CGG-ATC-ATC-AA-3; antisense, 5-CTG-GATGGA-CGT-GTA-GAA-GT-3) and G3PDH (983-bp; sense, 5-TGA-AGG-TCG-GGT-GTG-AAC-GGA-TTT-GGC-3; antisense, 5-CAT-GTA-GGC-CAT-GAG-GTC-CAC-CAC-3). The possible contamination of any PCR component was excluded by performing a PCR reaction with these components in the absence of RT product in each set of experiments (negative control). Twenty microliters of RT-PCR product was checked by using $2 \%$ agarose gel electrophoresis containing $0.2 \mu \mathrm{g} / \mathrm{mL}$ ethidium bromide, followed by photography under UV transillumination. Quantification of RNA transcripts was analyzed according to the method described previously with some modification. ${ }^{19}$ In brief, the levels of expression of the transcripts were normalized to that of G3PDH mRNA in the same tissue sample. The appropriate number of PCR cycles and the optimal combination of the amount of first-strand cDNAs were determined for each set of primers to ensure that all PCR reactions were carried out in the linear quantitative phase of the amplification process. The PCR products were size fractionated on agarose gels, visualized by means of ethidium bromide staining, and photographed. The photographs of the ethidium bromide-stained gels were scanned with an imaging densitometer linked to a personal computer equipped with image analysis software. The size and optical density of the bands produced by the PCR products were quantified by using the Kodak digital science 1-dimensional image analysis software (EDAS 120, Eastman Kodak Company). For statistical comparison, more than 3 independent samples in each experiment were performed to obtain the ratio of the density of Hsp70 to G3PDH mRNA levels. 


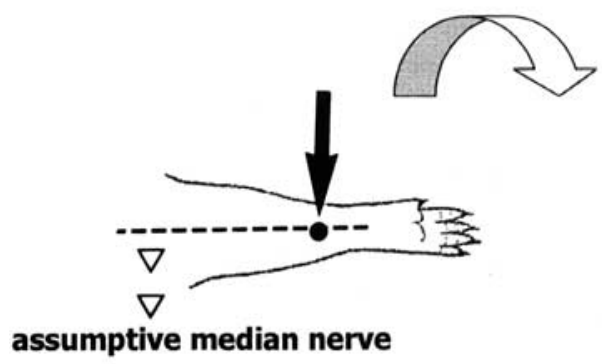

A

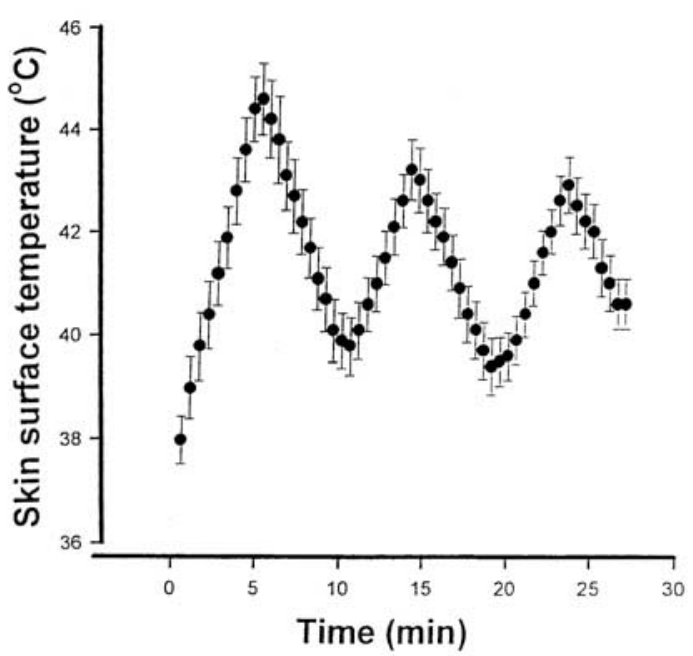

B

Figure 1. Experimental setup (A) and skin temperature curve obtained by means of LSTS (B). LSTS was applied above $(0.5 \mathrm{~cm})$ and to the skin area $1.5 \mathrm{~cm}$ proximal to the left palm crease. The dashed line indicates the assumptive median nerve. LSTS was brought about by means of intermittent turning on and off of the heat generator, as described in the "Methods" section.

\section{Local Heat Stress by LSTS}

Application of local heat stress compared with whole-body hyperthermia was achieved by means of LSTS on the skin area but no direct contact with the skin surface (Figure 1, A). The effect of LSTS has been reported in our laboratory to induce relaxation of the sphincter of Oddi in cats and rabbits, as well as the internal sphincter in rabbits. ${ }^{14-16}$ In brief, LSTS was achieved by application of a heat generator to and above $(0.5 \mathrm{~cm})$ the skin area, and a fluctuating skin temperature curve was obtained by intermittently turning on and off the switch of the heat generator (ie, 4 minutes on and 5 minutes off for 3 courses; Figure 1,B). Usually, it took 27 minutes to complete one cycle of LSTS. LSTS was applied to and above the left median nerve territory to study the myocardial expression of Hsp70, and the gene expression of myocardial Hsp70 was analyzed by means of Western blotting and RT-PCR. The left median nerve territory was located $1.5 \mathrm{~cm}$ proximal to left palm crease just above the median nerve.

\section{Preconditioning by LSTS}

After achievement of light anesthesia with ketamine $(50 \mathrm{mg} / \mathrm{kg}$ administered intraperitoneally), animals were randomly allocated to 2 preconditioning groups, namely the LSTS and placebo (without LSTS) groups. In the LSTS group parameters of preconditioning for thermotolerance study include 3 doses of LSTS on the left median nerve territory at an interval of 12 hours and intermittent heating (on for 4 minutes/off for 5 minutes) for 27 minutes. Subsequent experiments for ischemia-reperfusion injury of the heart were performed 12 hours after the third cycle of preconditioned LSTS.

\section{Animal Model and Parameters for Ischemia- Reperfusion Injury of the Heart}

Ischemia-reperfusion injury in a rat model was made as described previously. ${ }^{20,21}$ In brief, male Sprague-Dawley rats preconditioned with LSTS were anesthetized with urethane $(1.25 \mathrm{gm} / \mathrm{kg})$ administered intraperitoneally, and the trachea was cannulated for artificial respiration with a ventilator. Polyethylene catheters were cannulated into the femoral artery to monitor the blood pressure, and a standard lead-1 electrocardiogram (ECG) was recorded by attaching silver electrodes to the extremities of animals and monitored by a polygrapher (RS 2400, Gould). After the chest was opened by means of a left thoracotomy and the fourth and fifth ribs were sectioned, the pericardium was incised, followed by easing the heart out of the chest, with gentle pressure on the rib cage. A 6-0 silk suture was placed under the left main coronary artery, and the heart was repositioned in the chest for 15 minutes. Animals in which the procedure produced arrhythmia or death during the first 5 minutes were excluded from this study. The procedure whereby the silk was fashioned into a snare allowed occlusion of the blood supply to the left coronary artery for 30 minutes. The reperfusion was achieved by releasing the tension applied to the ligature for 10 minutes. Successful ligation of the coronary artery was validated by means of observation of a decrease in arterial pressure and ECG changes (increase in R-wave and ST-segment elevation), which is indicative of ischemia. Reperfusion was confirmed by means of an increase in arterial pressure, ECG changes (decrease of the R wave and normalization of the ST segment), and sudden occurrence of arrhythmia. Before and during the ischemia-reperfusion period, heart rate, blood pressure, and ECG changes were recorded on a computer equipped with an offline data analysis software (AcqKnowlege, Biopac System). Arrhythmia, such as ventricular fibrillation and premature ventricular beat, were evaluated as described previously. ${ }^{22}$ Serum creatine phosphokinase and creatine kinase isozyme (MB fraction) levels were assayed by means of electrophoresis to determine the degree of myocardial injury. 


\section{Mitochondria Preparation and Mitochondrial Functions}

Mitochondria were prepared from the hearts of male rats, as previously described by Vercesi and colleagues. ${ }^{23}$ Sprague-Dawley rats were killed by means of decapitation, and their hearts were quickly excised and thoroughly washed with ice-cold SEH buffer $(0.25 \mathrm{~mol} / \mathrm{L}$ sucrose, $0.5 \mathrm{mmol} / \mathrm{L}$ ethyleneglycol-bis-[ $\beta$-aminoethylether]-N,N,N,N, tetra-acetic acid, and $3 \mathrm{mmol} / \mathrm{L} \mathrm{N}$-2-hydroxyethylpiperazine-N-ethanesulfonic acid, $\mathrm{pH}$ 7.2). All the following procedures were performed at $4^{\circ} \mathrm{C}$. The hearts were finely minced with a pair of scissors in SEH buffer. The dispersed heart tissue was homogenized with a glass pestle and diluted with SEH and pronase $(0.1 \mathrm{mg} / \mathrm{mL} \mathrm{SEH}$ buffer $)$ solution to $10 \mathrm{~mL}$ per gram of heart tissue for 15 minutes. The hearts were washed in $0.25 \mathrm{~mol} / \mathrm{L}$ sucrose twice. The homogenates were centrifuged at $400 \mathrm{~g}$ for 5 minutes in the SS34 rotor of an RC-5C Sorvall refrigerated highspeed centrifuge (Du Pont). The supernatant was decanted and centrifuged at $700 \mathrm{~g}$ for 5 minutes. After recentrifugation of the supernatant at $2500 \mathrm{~g}$ for 10 minutes, the pellet was resuspended in SEH solution and centrifuged again for 10 minutes at $6000 \mathrm{~g}$. The mitochondria-rich red-brown lower layer was suspended in $0.5 \mathrm{~mL}$ of SEH solution per gram of heart tissue. Because the respiratory function of mitochondria decreased rapidly, all respiratory experiments were performed with the freshly prepared mitochondria.

\section{Determination of Respiratory Control Ratios}

The glutamate and malate solution-supported (Sigma) respiration rates of the mitochondria were determined polarographically in an oxygen monitor equipped with a Clark oxygen electrode (Gilson Medical Electronics). Usually, 0.4 to $0.8 \mathrm{mg}$ of freshly prepared mitochondria was incubated in the reaction chamber containing 1.6 $\mathrm{mL}$ of assay mixture before the addition of substrates. The addition of glutamate and malate solution induced slow oxygen uptake (state 4), and $400 \mathrm{nmol}$ of adenosine diphosphate was introduced to the assay system to stimulate respiration to reach an active state (state 3 ). States 3 and 4 of respiration were defined as described previously. ${ }^{24}$ The respiratory control ratio (RCR) was measured as the quotient of the respiration rate of state 3 to that of state 4 .

\section{Statistics}

Data were expressed as means \pm SEM. Significance was determined by using 1-way analysis of variance, followed by the Bonferroni-corrected $t$ test or the Wilcoxon rank sum test for stepwise comparison. For noncontinuous variables, the difference between groups was analyzed by using the Fisher exact test.

\section{Results}

\section{Dose Selection for Local Heat Stress (LSTS)}

To determine the optimal cycle of local heat stress or LSTS on myocardial Hsp70 expression, cycle-related experiments were performed. The results showed that there was an increase of myocardial expression of Hsp70 after different cycles of LSTS on the left median nerve territory, with the peak expression at 3 cycles of LSTS (Figure 2).

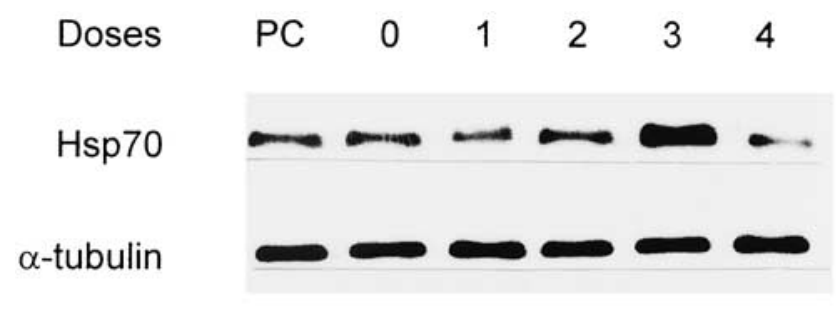

Figure 2. Dose response of Hsp70 expression after LSTS on the right lower abdominal wall. The expression of Hsp70 in the muscle beneath the LSTS skin area was analyzed by means of Western blotting, as described in the "Methods" section.

\section{Effects of LSTS on the Left Median Nerve Territory on Hsp70 Expression in Regional Tissue}

The Hsp70 expression in regional muscle near the left median nerve just beneath the skin area undergoing LSTS and the remote organs (liver and heart) were examined to study the effect of LSTS on different tissues. The results showed that Hsp70 expression in the muscles near the left median nerve or LSTS $(+)$ site was significantly higher than that of the right side or $\operatorname{LSTS}(-)$ site in the same animals (n $=3, P<.05$, Wilcoxon sign rank test; Figure 3 ).

\section{Effects of LSTS on the Left Median Nerve Territory on Hsp70 Expression in Remote Organs}

Myocardial and hepatic Hsp70 expression were analyzed by means of Western blotting to elucidate the specificity of LSTS. The results showed a significant increase of Hsp70 expression in the hearts from $\operatorname{LSTS}(+)$ rats compared with in those from $\operatorname{LSTS}(-)$ rats $(\mathrm{n}=7 ; P<.05$, Wilcoxon rank sum test), whereas no significant change on hepatic Hsp70 expression was noticed between the $\operatorname{LSTS}(+)$ and $\operatorname{LSTS}(-)$ groups when the local heat stress was applied to the left median nerve territory (Figure 4). When analyzed with RT-PCR, the results demonstrated an upregulated Hsp70 transcription in the hearts from $\operatorname{LSTS}(+)$ rats (Hsp70/ $\mathrm{G} 3 \mathrm{PDH}$ ratio $=0.30 \pm 0.012$ ) compared with that from $\operatorname{LSTS}(-)$ rats $(\mathrm{Hsp} 70 / \mathrm{G} 3 \mathrm{PDH}$ ratio $=0.10 \pm 0.015 ; P<$ .05 , Wilcoxon rank sum test; Figure $5, A$ ). There was no significant change on hepatic Hsp70 gene expression between the LSTS $(+)$ group $(\mathrm{Hsp} 70 / \mathrm{G} 3 \mathrm{PDH}$ ratio $=0.15 \pm$ $0.010)$ and the $\operatorname{LSTS}(-)$ group $(\mathrm{Hsp} 70 / \mathrm{G} 3 \mathrm{PDH}$ ratio $=$ $0.13 \pm 0.008 ; P>.05$, Wilcoxon rank sum test; Figure 5, $B$ and $C$ ). The data demonstrate the organ specificity of LSTS on the left median nerve territory.

\section{Preconditioning Animals With LSTS on the Left Median Nerve Territory Protects the Myocardium From Subsequent Ischemia-Reperfusion Injury} LSTS was applied to and above the left median nerve territory for 3 cycles to study the modulatory effect of preconditioned LSTS on myocardial ischemia-reperfusion 

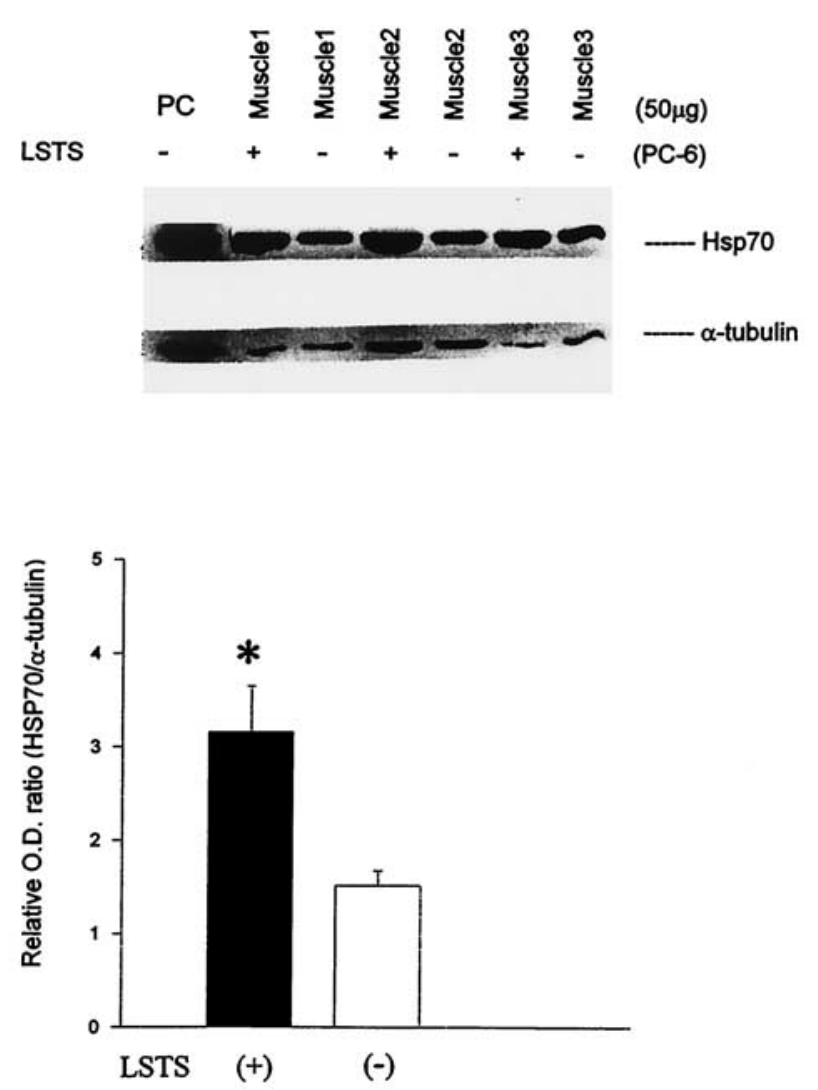

Figure 3. The expression of Hsp70 in the muscle near the left median nerve territory after 3 cycles of LSTS. The expression of Hsp70 in the muscle underlying the left median nerve was analyzed by means of Western blotting, as described in the "Methods" section. The asterisk indicates statistical significance $(P<$ 0.05 ) between the LSTS(+) group (filled column) and the LSTS(-) group (open column). OD, Optical density.

injury. Subsequent ischemia-reperfusion injury of the heart were performed 12 hours after the third cycle of preconditioned LSTS. Severe arrhythmia (ventricular tachycardia and ventricular fibrillation) was observed in control animals (no LSTS preconditioning; Figure 6, A), whereas less arrhythmia, in terms of duration of ventricular tachycardia and ventricular fibrillation, was observed in the LSTS group (Figure 6, B). The LSTS(+) group had a lower mortality rate and lower incidence of ventricular arrhythmia compared with those of the LSTS $(-)$ group $(P<.05$, Fisher exact test). The duration of arrhythmia showed significant change between the $\operatorname{LSTS}(+)$ and $\operatorname{LSTS}(-)$ groups after the ischemia-reperfusion procedure $(P<.05$, Wilcoxon rank sum test). There was a significant decrease of serum cardiac enzymes (creatine phosphokinase and creatine kinase MB) in the LSTS(+) group compared with in the LSTS(-) group. In addition, rats preconditioned with LSTS had better myocardial mitochondrial function in terms of the
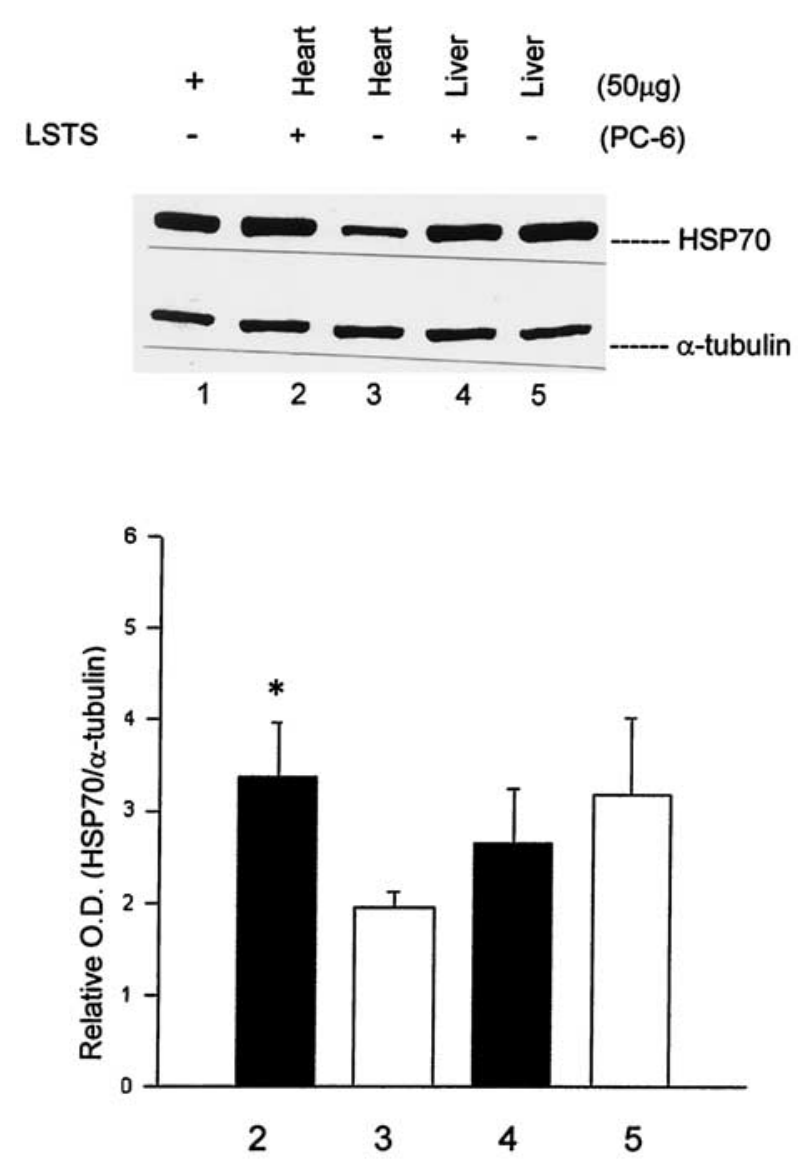

Figure 4. The expression of Hsp70 in the heart and liver after 3 cycles of LSTS on the left median nerve territory. The expression of Hsp70 in the heart and liver tissues was analyzed by means of Western blotting, as described in the "Methods" section. The asterisk indicates statistical significance $(P<.05)$ between the LSTS(+) group (filled columns) and the LSTS(-) group (open columns). OD, Optical density.

RCR, with an RCR of $5.42 \pm 0.05$ compared with that seen in $\operatorname{LSTS}(-)$ rats $(\mathrm{RCR}=4.76 \pm 0.14 ; P<.05$, Wilcoxon rank sum test; Figure 7). Taken together, the results suggest that LSTS preconditioning on the left median nerve territory attenuates myocardial damage from subsequent ischemiareperfusion injury.

\section{Discussion}

In the present study we clearly demonstrated that LSTS on the left median nerve territory upregulated the gene expression of Hsp70 in the heart, which is organ specific. We also found that preconditioning with LSTS protected the rat heart against ischemia-reperfusion injury. To our knowledge, these are novel findings that Hsp70 gene expression can be regulated by local heat stress (not whole-body hyperthermia) through a somatovisceral reflex.

Among the systemic consequences of reperfusion, lipid 


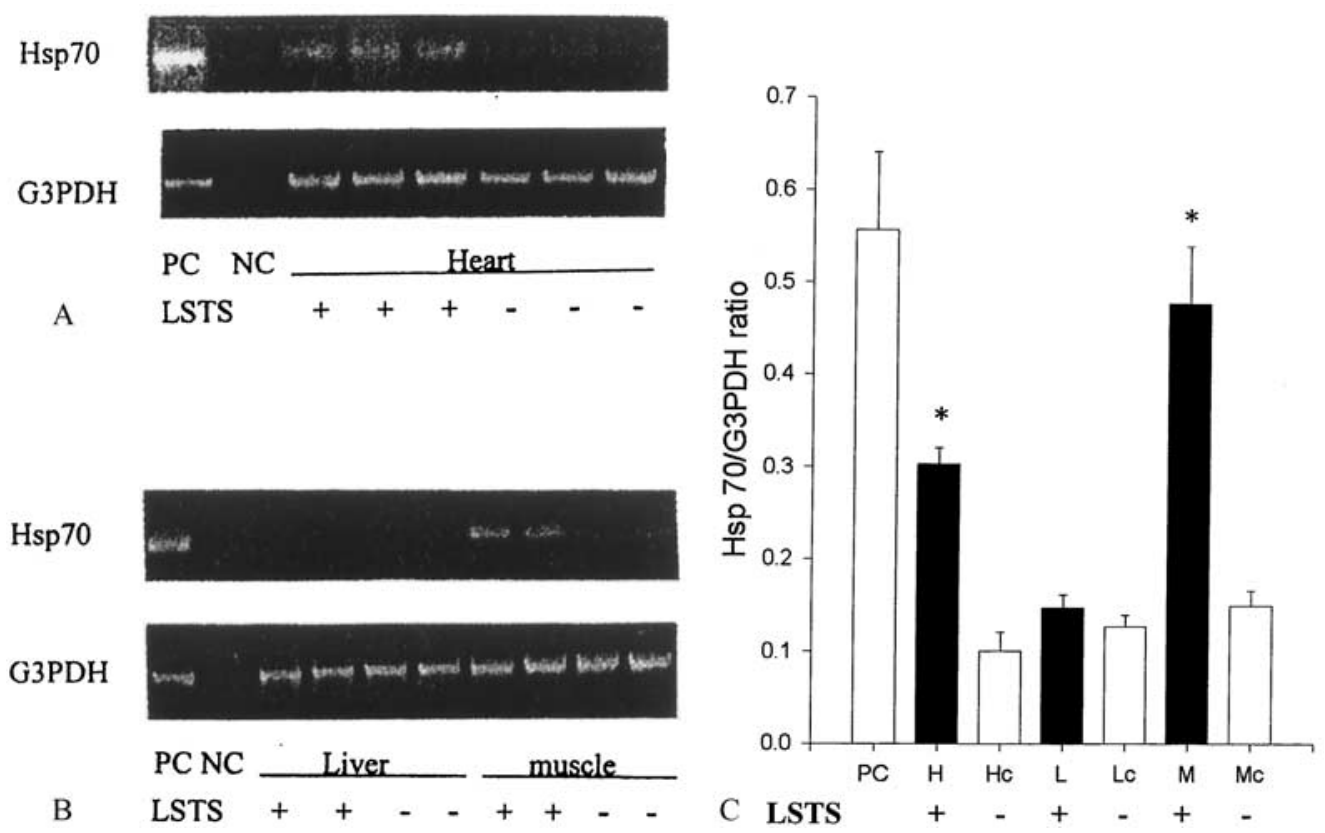

Figure 5. RT-PCR analysis of Hsp70 in the heart (A) and liver and muscle (B) after 3 cycles of LSTS on the left median nerve territory. For the primer sequences of Hsp70, reactions of RT-PCR were described in the "Methods" section. The density of Hsp70 to G3PDH mRNA levels were analyzed by means of computerized densitometry and expressed as the Hsp70/G3PDH ratio (C). The data were reproduced from more than 3 independent experiments. The asterisk indicates statistical significance between the LSTS(+) group (filled columns) and the LSTS(-) group (open columns). $H, L$, and $M$ indicate heart, liver, and muscle tissues in the LSTS group, respectively; whereas $H c, L c$, and $M c$ indicate heart, liver, and muscle tissues in the control or LSTS(-) group, respectively. PC, Positive control.

peroxidation is probably the most damaging effect of free radicals. Endogenous free radicals generated during ischemia-reperfusion injury induce damage to the cytoskeleton and disruption of microtubules and result in mitochondrial swelling and loss of mitochondrial functions. ${ }^{25,26}$ Respiratory functions are very sensitive to minor membrane damage. ${ }^{27}$ Any interruption of mitochondrial membrane integrity leads to proton leakage, uncoupling of the electron transport chain, oxidative phosphorylation, cellular dysfunction, and death. ${ }^{28}$ Although the heat shock or stress response refers to the progress whereby stressful stimuli (eg, hyperthermia) induce a transient resistance of the cells to subsequent noxious stimuli, it remains controversial between the expression of $\mathrm{Hsp} 70$ and its cardioprotective function in many studies. For example, some studies have suggested that overexpressed Hsp70 plays a direct role in the enhancement of myocardial tolerance against ischemiareperfusion injury, ${ }^{6-9}$ whereas others have presented differing opinions. ${ }^{29}$ Our results showed that there was an increase of myocardial Hsp70 gene expression and improved myocardial functions in terms of durations of arrhythmia, mortality rates, and mitochondrial functions when animals were preconditioned with LSTS on the left median nerve territory. Because no Hsp70 gene knockout or gene transfer experiments were done in our system to provide direct evidence of the protective role of Hsp70, the direct causeand-effect relationship requires further elucidation.

There is consensus that it is not feasible to perform whole-body hyperthermia in human subjects, although it might induce Hsp70 expression in the heart. In addition, it is impractical to perform whole-body hyperthermia preconditioning in human subjects. On the contrary, LSTS is an alternative to local heat stress, which can be easily applied on the skin area of the human body. In our system the rate of increase of the temperature varied if the distance between the heating rod and the skin changed. We found that LSTS at a distance of $0.5 \mathrm{~cm}$ and a stimulated area of $1 \mathrm{~cm}$ (diameter) was the optimal condition to induce myocardial Hsps (data not shown). Our previous works showed that temperatures ranging from $41^{\circ} \mathrm{C}$ to $43^{\circ} \mathrm{C}$ are critical points in modulating many visceral functions, which is consistent with previous studies on inducing Hsp expression. ${ }^{10,14-16}$ It is noteworthy that the fluctuating curve of temperature on LSTS (Figure 1, $B$ ) is designed to make the temperature increase and decrease from the critical point of $41^{\circ} \mathrm{C}$ so that the heat-sensitive neural transmission would not be tolerated. In addition, in such mild heat stimulation, no skin damage, such as burn injury or median nerve injury, was noticed.

In contrast to whole-body heat stress (hyperthermia), 


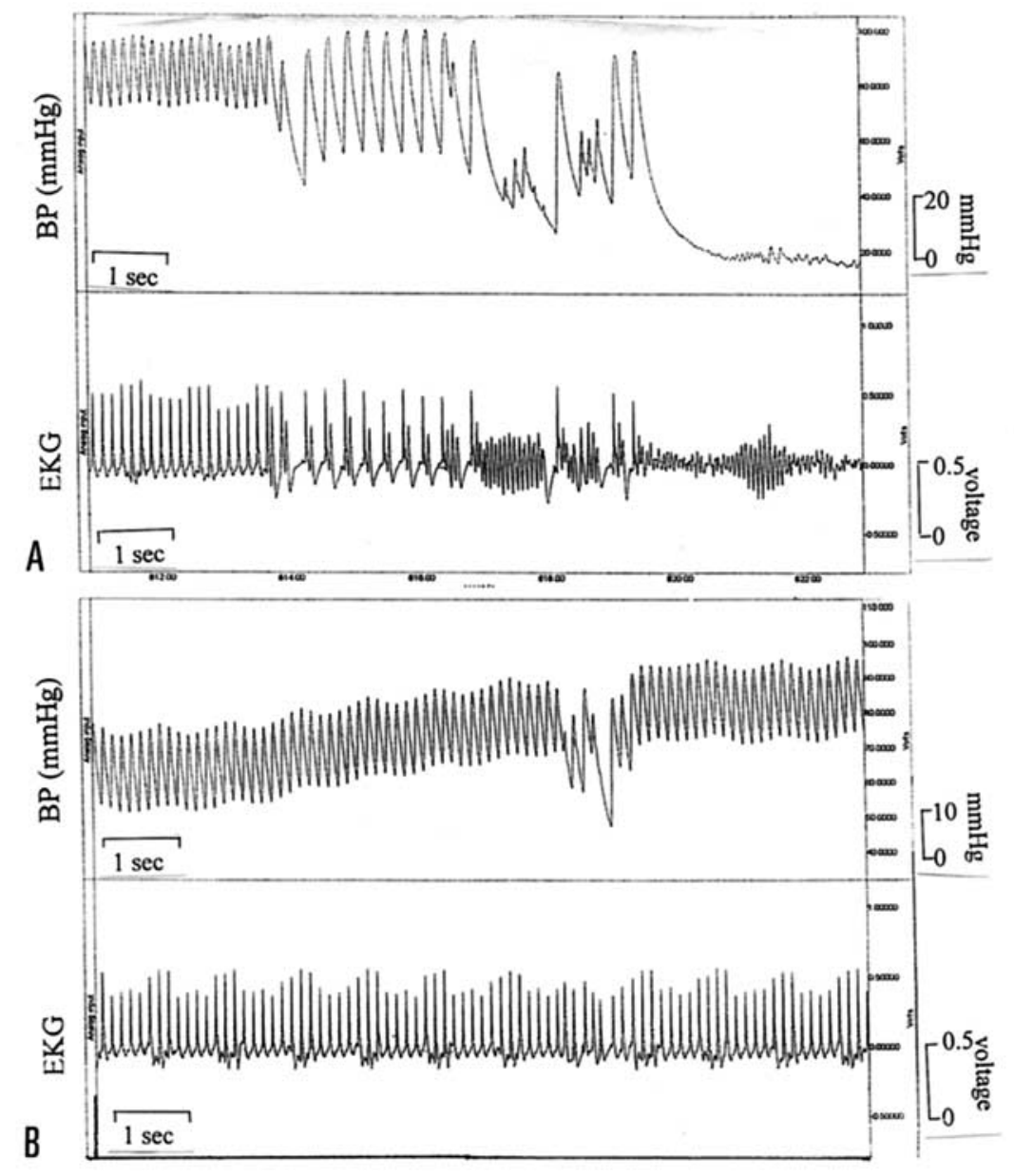

Figure 6. ECG and blood pressure monitoring during ischemia-reperfusion injury in rats preconditioned with $(A)$ and without (B) LSTS on the left median nerve territory. The changes in blood pressure (upper panel) and ECG results (lower panel) in 2 groups receiving ischemia-reperfusion injury was recorded. BP, Blood pressure.

LSTS is a kind of local heat stress. Although there was an increased expression of Hsp70 in the muscle underlying the LSTS area, this was not the case for hepatic Hsp70 expression when LSTS was applied to the left median nerve territory. Furthermore, our previous study showed that LSTS on the right seventh intercostal nerve territories increases hepatic, but not myocardial, Hsp70 expression. The results also demonstrated that sublethal preconditioning of rats with LSTS on the right seventh intercostal nerve territory increases Hsp70 expression and decreases malondialdehyde formation in subsequent ischemia-reperfusion injury in the liver. ${ }^{16}$ These facts make the possibility that the myocardial responses to LSTS on the left median nerve territory are only reflective of a global whole-body response less likely. On the contrary, the visceral responses evoked by LSTS on skin area are organ specific. With regard to the result that there was more abundant expression of myocardial Hsp70 after 3 cycles than after 4 cycles of LSTS on the left median nerve territory (Figure 2), we attributed this phenomenon to the possibilities of more degradation of Hsp70 or a plateau effect of 4 cycles of LSTS. In addition, the increase of relative abundance of myocardial Hsp70 mRNA after LSTS on the left median nerve territory might be caused by an increase in gene expression, an increase in mRNA stability, or both. Nuclear run-on studies are under investigation.

With regard to the potential of clinical implication of LSTS, we have applied a heat-generating pad instead of a heating rod in animal studies to relax the hypertonic anal sphincter in human subjects. ${ }^{30}$ The effect of LSTS is postulated to be through the activation of heat-sensitive neural release of nitric oxide. ${ }^{15}$

In summary, preconditioned LSTS on the left median nerve territory has a beneficial effect in protecting the rat heart against ischemia-reperfusion injury. This is the advantage compared with pretreatment by means of whole-body 


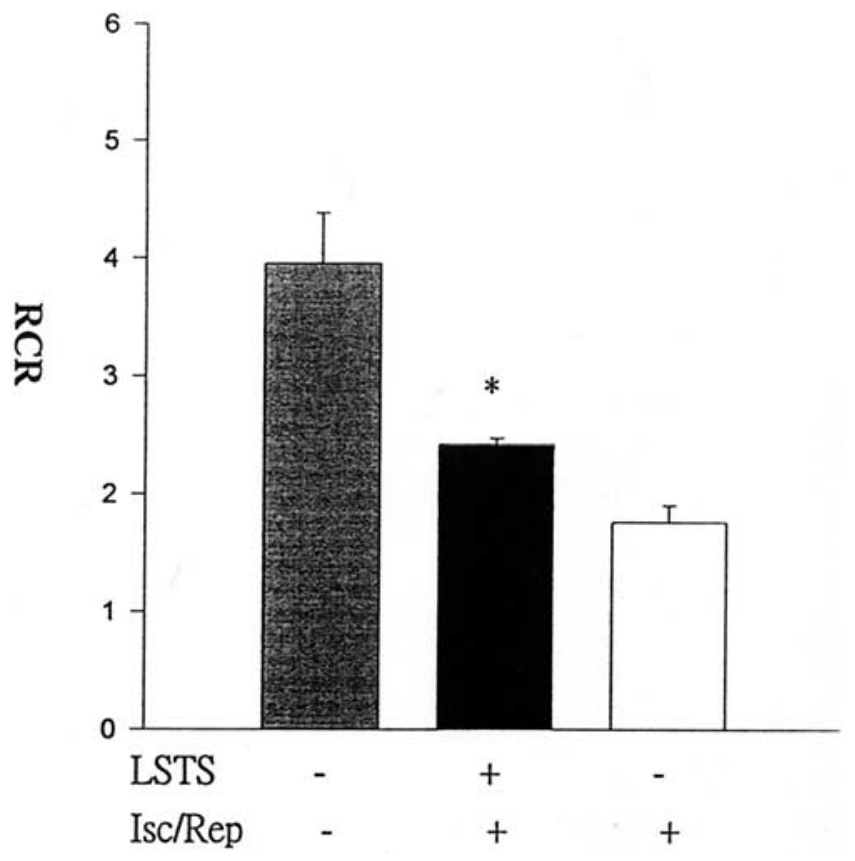

Figure 7. RCRs of heart mitochondria were obtained by means of analysis of 3 independent experiments. The asterisk indicates statistical significance $(P<.05)$ between the LSTS $(+)$ group (filled column) and the LSTS(-) group (open column) during 10 minutes of ischemia and $\mathbf{1 0}$ minutes of reperfusion. The normal control group is shown in the shaded column. Isc/Rep, Ischemia and reperfusion.

hyperthermia or brief myocardial ischemia. LSTS is an easily applicable alternative and will bring into perspective the clinical prevention of ischemic heart disease or heart transplantation.

We thank Miss Yi-Hong Hsieh for her excellent technical assist.

\section{References}

1. Bukau B, Horwich AL. The Hsp70 and Hsp60 chaperone machines. Cell. 1998;92:351-66.

2. Jaattela M. Heat shock proteins as cellular life guards. Ann Med. 1999;31:261-71.

3. Mosser DD, Caron AW, Bourget L, Denis-Larose C, Massie B. Role of human heat shock protein hsp70 in protection against stress-induced apoptosis. Mol Cell Biol. 1997;17:5317-27.

4. Akagawa H, Ishii A, Mizuno S. Suppression of thermotolerance development through cycloheximide-induced negative control of stress protein gene expression. J Biochem. 1998;123:226-32.

5. Hutter MM, Sievers RE, Barbosa V, Wolfe CL. Heat-shock protein induction in rat hearts. A direct correlation between the amount of heat-shock protein induced the degree of myocardial protection. Circulation. 1994;89:355-60.

6. Marber MS, Mestril R, Chi SH, Sayen R, Yellon DM, Dillmann WH. Overexpression of the rat inducible $70 \mathrm{KD}$ heat stress protein in a transgenic mouse increase the resistance of the heart to ischemic injury. J Clin Invest. 1995;95:1446-56.

7. Plumier JCL, Ross BM, Currie RW, Angelidis CE, Kazlaris H, Kollias $\mathrm{G}$, et al. Transgenic mice expressing the human heat shock protein 70 have improved post-ischemic myocardial recovery. J Clin Invest. 1995;95:1854-60.

8. Brar BK, Stephanou A, Wagstaff MJ, Coffin RS, Marber MS, Engelmann G, et al. Heat shock proteins delivered with a virus vector can protect cardiac cells against apoptosis as well as against thermal or hypoxic stress. J Mol Cellular Cardiol. 1999;31:135-46.

9. Suzuki K, Sawa Y, Kaneda Y, Ichikawa H, Shirakura R, Matsuda H. In vivo gene transfection with heat shock protein 70 enhances myocardial tolerance to ischemia- reperfusion injury in rat. J Clin Invest. 1997;99:1645-50.

10. Lin MT. Heatshock-induced cerebral ischemia and neuronal damage: involvement of cytokines and monoamines. Ann N Y Acad Sci. 1997; 813:572-80.

11. Chao DM, Shen LL, Tjen-A-Looi S, Pitsillides KF, Li P, Longhurst JC. Naloxone reverse inhibitory effect of electroacupuncture on sympathetic cardiovascular reflex response. Am J Physiol. 1999;276:2127-34.

12. Cao Q, Lin J, Chen S, Han Z. Effect of electroacupuncture at neiguan on myocardial microcirculation in rabbits with acute myocardial ischemia. J Tradit Chin Med. 1998;18:134-9.

13. Li P, Pitsillides KF, Rendig SV, Pan HL, Longhurst JC. Reversal of reflex-induced myocardial ischemia by medial nerve stimulation, a feline model of electroacupuncture. Circulation. 1998;97:1186-94.

14. Chiu JH, Lui WY, Chen YL, Hong CY. Local somatothermal stimulation inhibits sphincter of Oddi motility in cats, rabbits, and humans through the neural release of nitric oxide. Life Sci. 1998;63:413-28.

15. Jiang JK, Chiu JH, Lin JK. Local thermal stimulation inhibits the motility of internal anal sphincter through nitrergic neural release of nitric oxide. Dis Colon Rectum. 2000;43:381-8.

16. Lin YH, Chiu JH, Tung HH, Tsou MT, Lui WY, Wu CW. Preconditioned somatothermal stimulation on right 7 th intercostal nerve territory increases hepatic heat shock protein 70 and protects the liver from ischemia-reperfusion injury in rats. J Surg Res. 2001;99:328-34.

17. Bradford M. A rapid and sensitive method for quantitation of microgram quantities of protein utilizing the principle of protein-dye binding. Anal Chem. 1976;72:248-54.

18. Chomczynski P, Sacchi N. Single-step method of RNA isolation by acid guanidinium thiocyanate-phenol-chloroform extraction. Anal Biochem. 1987;162:156-9.

19. Wasser S, Ho JMS, Ang HK, Tan CEL. Salvia miltiorrhiza reduces experimentally-induced hepatic fibrosis in rats. J Hepatol. 1998;29: 760-71.

20. Tsai SK, Huang SS, Hong CY. Myocardial protective effect of honokiol: an active component in Magnolia officinalis. Planta Med. 1996;62:503-6.

21. Hong CY, Huang SS, Tsai SK. Magnolol reduces infarct size and suppresses ventricular arrhythmia in rats subjected to coronary ligation. Clin Exp Pharmacol. 1996;23:660-4.

22. Heimburger RF. Injection into pericardial sac and ligation of coronary artery of rat. Arch Surg. 1946;52:677-89.

23. Vercesi A, Reynafarje B, Lehninger AL. Stoichiometry of $\mathrm{H}^{+}$ejection and $\mathrm{Ca}^{2+}$ uptake couples to electron transport in rat heart mitochondria. J Biol Chem. 1978;253:6379-85.

24. Chance B, William GR. The respiratory chain and oxidative phosphorylation. Adv Enzymol. 1956;17:65.

25. Patriarca EJ, Kobayashi GS, Maresca B. Mitochondrial activity and heat-shock response during morphogenesis in the pathogenic fungus Histoplasma capsulatum. Biochem Cell Biol. 1992;70:207-14.

26. Patriarca EJ, Maresca B. Acquired thermotolerance following heat shock protein synthesis prevents impairment of mitochondrial ATPase activity at elevated temperatures in Saccharomyces cerevisiae. Exp Cell Res. 1990;190:57-64.

27. Gronzalez-Flecha B, Cutrin JC, Boveris A. Time course and mechanism of oxidative stress and tissue damage in rat liver subjected to in vivo ischemia-reperfusion. J Clin Invest. 1993;91:456-64.

28. Grace PA. Ischemia-reperfusion injury. Br J Surg. 1994;81:637-47.

29. Su CY, Chong KY, Chen J, Ryter S, Khardori R, Lai CC. A physiologically relevant hyperthermia selectively activates constitutive hsp70 in $\mathrm{H} 9 \mathrm{c} 2$ cardiac myoblasts and confers oxidative protection. $J$ Mol Cell Cardiol. 1999;31:845-55.

30. Jiang JK, Chiu JH, Lin JK. Local thermal stimulation relaxes hypertonic anal sphincter: evidence of somatoanal reflex. Dis Colon Rectum 1999;42:1152-9. 